\title{
Effect of the recovery duration of a repeated sprint exercise on the power output, jumping performance and lactate concentration in pre- pubescent soccer players
}

\author{
Pantelis T. Nikolaidis ${ }^{1}$, Beat Knechtle ${ }^{2}$ \\ ${ }^{1}$ Exercise Physiology Laboratory, Nikaia, Greece; ${ }^{2}$ Instutute of Primary Care, University of Zurich, Switzerland
}

\section{Summary}

Study aim: The aim of the present study was to examine the effect of two different recovery durations ( 2 min versus 5 min) on the physiological responses (power output, stretch-shortening cycle and lactate concentration) to a $5 \times 6 \mathrm{~s}$ repeated cycling sprint exercise protocol in pre-pubescent soccer players.

Materials and methods: Twelve male soccer players (age $12.23 \pm 0.55 \mathrm{yrs}$, body mass $43.6 \pm 5.5 \mathrm{~kg}$ and height $156.1 \pm 5.8 \mathrm{~cm}$ ) performed $5 \times 6 \mathrm{~s}$ sprints on a cycle ergometer (Ergomedic 874E, Monark, Sweden) against 0.075 times their body mass resistance on two occasions within a week. In one session there was a 2 min recovery and in the other there was a 5 min recovery in a counterbalanced order. A squat jump (SJ) and a countermovement jump (CMJ) were tested before and after each trial, and the eccentric utilisation ratio (EUR) was calculated as $\mathrm{CMJ} / \mathrm{SJ}$.

Results: No significant trial $\times$ recovery interaction was observed in the participants' peak power $\left(\mathrm{p}=0.891, \eta^{2}=0.118\right)$, mean power $\left(p=0.910, \eta^{2}=0.106\right), S J\left(p=0.144, \eta^{2}=0.630\right)$, CMJ $\left(p=0.616, \eta^{2}=0.347\right)$ and EUR $\left(p=0.712, \eta^{2}=0.295\right)$. However, a main effect of the trial on the CMJ of a large magnitude $\left(p=0.006, \eta^{2}=0.862\right)$ was found, in which a higher score was recorded in the third trial than in the first trial $(23.3$ versus $21.8 \mathrm{~cm})$. No differences were found in the lactate concentrations examined $5 \mathrm{~min}$ after the end of the protocol between the two recovery conditions $\left(6.7 \pm 1.8 \mathrm{vs} .6 .0 \pm 1.6 \mathrm{mmol} \cdot \mathrm{L}^{-1}\right.$, in the 2 and 5 min recovery, respectively, Cohen's d $=0.4$ ).

Conclusions: The duration of the passive recovery time ( $2 \mathrm{~min}$ vs. $5 \mathrm{~min}$ ) in trials of repeated sprints did not induce important changes either to the indices of the jumping performance or to the power output in pre-pubescent participants.

\section{Keywords: Anaerobic metabolism - Countermovement jump - Maximal exercise - Recovery - Squat jump}

\section{Introduction}

Repeated sprint exercise (RSE) has been recommended as an optimal training tool to improve the repeated sprinting ability, which is a physical fitness component that has been recognised during the last decades as playing a key role in the performance of team sports (e.g. soccer, basketball and handball) $[23,31]$. In addition to its beneficial role for the performance of team sports, RSE has recently been shown to ameliorate health-rated parameters such as cardio-metabolic risk factors and psychological health $[26,30,34]$. Hence, the optimal prescription of RSE impacts both the sport performance and the health of an individual. Among the variables that coaches can manipulate in RSE (mode of exercise, workload, mode of recovery, number and duration of trials), an important variable is the duration of the recovery between the trials [3, 16, 24, 27].

The duration of the recovery in RSE using cycling has ranged from $24 \mathrm{~s}$ [16] to $20 \mathrm{~min}$ [1]. For instance, a $24 \mathrm{~s}$ recovery has been used in protocols of $5 \times 6 \mathrm{~s}$ (number of trials $\times$ duration of each trial) [16]; a 20 and 90 s recovery has been used in $12 \times 4 \mathrm{~s}$ [18]; a 25, 50 and $100 \mathrm{~s}$ recovery in $10 \times 5 \mathrm{~s}$ [24]; a $30 \mathrm{~s}$ recovery in $4 \times 10 \mathrm{~s}$ [21], $5 \times 6 \mathrm{~s}$ [9] and $6 \times 6 \mathrm{~s}$ protocols [8]; $30 \mathrm{~s}, 1$ and 5 min recovery in $10 \times 10 \mathrm{~s}$ [27]; and a $40 \mathrm{~s}$ recovery in a $10 \times 10 \mathrm{~s}$ protocol [17]. Moreover, longer recovery times have ranged from $1.5,3$ and $6 \mathrm{~min}$ in $2 \times 30 \mathrm{~s}$ [3]; $3 \mathrm{~min}$ in $10 \times 10 \mathrm{~s}$ [25]; $4 \mathrm{~min}$ in $3 \times 30 \mathrm{~s}$ [32] and in $6 \times 30 \mathrm{~s}$ protocols [20]; $5 \mathrm{~min}$ in $5 \times 60 \mathrm{~s}$ [7]; and $20 \mathrm{~min}$ in a $3 \times 30 \mathrm{~s}$ protocol [1]. 
Observing the above-mentioned RSE protocols, a trend that should be highlighted is the use of a relatively large recovery time ( $>1 \mathrm{~min}$ ) mostly when the trial lasts for at least $30 \mathrm{~s}$. During these exercises, fatigue is recorded as changes in the power output, the blood lactate concentration and the knee extension maximum voluntary contraction force [18, 24, 25].

This analysis of previous studies clearly indicates a gap in the existing literature concerning the physiological impact of RSE using trials of a short duration (e.g. $6 \mathrm{~s}$ ) and a recovery of a long duration (e.g. $>1 \mathrm{~min}$ ). Moreover, most of the abovementioned studies have been conducted on adult participants. It has been shown that lactate responses to RSE might vary between men and boys, with the latter showing higher values of lactate concentrations after preforming the same exercise than the former $[12,28]$. This age-related difference in the lactate response to RSE indicates that boys need a longer recovery than adults. Knowledge about the differences in the physiological impact of the recovery could help sport scientists and practitioners (e.g. fitness trainers) working with children to prescribe an optimal exercise intensity by altering the recovery duration. Therefore, the aim of the present study was to examine the effect of two different recovery durations ( 2 min versus $5 \mathrm{~min}$ ) on the physiological responses (power output, jumping ability and lactate concentration) to a $5 \times 6 \mathrm{~s}$ RSE protocol in pre-pubescent soccer players. We hypothesised that the two recovery conditions would result in similar physiological effects.

\section{Materials and methods}

\section{Participants and study design}

Twelve pre-pubescent male soccer players (age $12.23 \pm 0.55$ yrs, Table 1) participated in the present study. The participants were members of an elite soccer academy in Athens, who each week practiced in three training sessions, each lasting for $90 \mathrm{~min}$, and competed in an official match. This was a convenience sample. The exclusion criteria were the presence of any illness or injury during

Table 1. Anthropometric characteristics of participants $(\mathrm{n}=12)$

\begin{tabular}{lcc}
\hline & Mean \pm SD & Range \\
\hline CA $(\mathrm{yrs})$ & $12.23 \pm 0.55$ & $11.47 ; 12.97$ \\
$\Delta$ APHV $(\mathrm{yrs})$ & $-2.26 \pm 0.59$ & $-2.94 ;-1.34$ \\
Body mass $(\mathrm{kg})$ & $43.6 \pm 5.5$ & $37.0 ; 54.5$ \\
Height $(\mathrm{cm})$ & $156.1 \pm 5.8$ & $149.0 ; 167.3$ \\
\hline
\end{tabular}

$\mathrm{CA}$ - chronological age, $\triangle \mathrm{APHV}$ - difference between CA and age at peak height velocity; range refers to minimal and maximal value the testing period; whereas the inclusion criteria were the participation in all of the soccer academy's activities (training sessions and matches). All of the participants were informed about the aim and the procedures of this study, and their parents provided informed consent. The local institutional review board approved this study. The study was conducted in March 2013, and included two testing sessions at the same time of day and under similar environmental conditions (temperature and humidity) separated by $48 \mathrm{~h}$. In the first session, the participants were measured for their anthropometric characteristics (body mass, height and skinfolds). At both sessions, they performed a repeated sprint ability protocol on a cycle ergometer: one session with a 2 min recovery; and the other with a 5 min recovery in a counterbalanced order. A squat jump (SJ) and a countermovement jump (CMJ) were tested before and after each trial, and the eccentric utilisation ratio (EUR) was calculated as $\mathrm{CMJ} / \mathrm{SJ}$. The lactate concentration in the blood was also measured after each repeated sprint ability protocol.

\section{Equipment and protocols}

The height and body mass were measured using a stadiometer (SECA, Leicester, UK) and an electronic scale (HD-351, Tanita, Illinois, USA) with the participants in minimal clothing. In addition to the standing height, the sitting height was measured with the same apparatus. The warm-up was similar in the two test sessions and consisted of $10 \mathrm{~min}$ of cycling against $1 \mathrm{~W} \cdot \mathrm{kg}^{-1}$ and 5 min of stretching exercises. The chronological age (CA) for each participant was calculated using a table of decimals for the year [29]. The peak height velocity (PHV), which reflects the maximum velocity in the growth of height, was used as an indicator of each boy's biological maturity. The age at PHV (APHV) was predicted by an equation taking into account the sex, date of birth, date of the measurement, height, sitting height and body mass [22]; and the difference ( $\triangle \mathrm{APHV}$ ) between $\mathrm{CA}$ and APHV was used as a measure of the biological age.

The RSE protocol consisted of five trials of maximal sprints, with each lasting for $6 \mathrm{~s}$, on a cycle ergometer $(E r-$ gomedic $874 E$, Monark, Sweden) against a braking force 0.075 times their body mass. In one session there was a $2 \mathrm{~min}$ recovery and in the other there was a $5 \mathrm{~min}$ recovery in a counterbalanced order. The braking force was that which was recommended for children of the participants' age in the present study to elicit the highest peak power $[6,10]$. The seat height was adjusted according to the participants' preference. The participants assumed an upright position on the cycle ergometer. Toe clips were used to secure their feet on the pedals. Two indices of performance were used for each sprint: the peak power (Ppeak), i.e. the highest power achieved over any $3 \mathrm{~s}$ interval within the $6 \mathrm{~s}$ exercise trial; and the mean power (Pmean), i.e. the 
average power achieved over the $6 \mathrm{~s}$ exercise trial [11]. Before the RSE protocol, the participants performed two trials for each of the jumping tests ( $\mathrm{SJ}$ and $\mathrm{CMJ}$ ) and the best score of the two trials was recorded. After each sprint protocol, they performed another trial of the SJ and CMJ. The height of each jump in the SJ and CMJ tests was estimated by using the Opto-jump system (Microgate Engineering, Bolzano, Italy) and was expressed in $\mathrm{cm}$. Briefly, the Opto-jump system measures the flight time of a jump and then uses this time to calculate the height of the jump [19]. In the SJ test, an initial semi-squatting position was assumed for $3 \mathrm{~s}$, and the hands were held on the waist during the jump [13]. The same position of the hands was assumed in the CMJ; however, in this case the initial position was upright and the participants performed a countermovement where they lowered to approximately a semi-squatting position and then jumped. The lactate concentration in the blood was assessed through a $20 \mu \mathrm{L}$ sample obtained from the finger, $5 \mathrm{~min}$ after the end of the RSE protocol, using the Accutrend Plus analyser (Roche Diagnostics, Basel, Switzerland).

\section{Statistical analysis}

The statistical analyses were performed using IBM SPSS v.20.0 (SPSS, Chicago, USA). The data was expressed as the mean and as the standard deviations of the mean (SD). A two-way repeated measures analysis of variance (ANOVA) examined the effects of the recovery duration ( 2 min versus $5 \mathrm{~min}$ ) in the trial on the Ppeak, Pmean, SJ, CMJ and EUR, where the within-subjects factors were the trial and the recovery. Subsequent comparisons between the trials were carried out using the post-hoc Bonferroni test. The magnitude of the differences among the splits was examined using the effect size eta squared $\left(\eta^{2}\right)$ and was evaluated as follows: small $\left(0.010<\eta^{2} \leq 0.059\right)$; moderate $\left(0.059<\eta^{2} \leq 0.138\right)$; and large $\left(\eta^{2}>0.138\right)$ [33]. The blood lactate concentration was compared between the two protocols using the dependent $t$-test. The effect size of the magnitude of their difference was evaluated by using Cohen's $d$ with the following criteria: $\mathrm{ES} \leq 0.2$, trivial; $0.2<\mathrm{ES} \leq 0.6$, small; $0.6<\mathrm{ES} \leq 1.2$, moderate; $1.2<$ ES $\leq 2.0$, large; and ES $>2.0$, very large [33]. The significance level was set at alpha $=0.05$.

\section{Results}

No significant trial $\times$ recovery interaction was observed in the Ppeak $\left(p=0.891, \eta^{2}=0.118\right)$, Pmean $(p=0.910$, $\left.\eta^{2}=0.106\right), S J\left(p=0.144, \eta^{2}=0.630\right), C M J(p=0.616$, $\left.\eta^{2}=0.347\right)$ and the EUR $\left(p=0.712, \eta^{2}=0.295\right)($ Fig. 1). However, a main effect of the trial on the height of the CMJ of a large magnitude ( $\left.p=0.006, \eta^{2}=0.862\right)$ was found, in which a higher score was recorded in the third trial than in the first trial $(23.3$ versus $21.8 \mathrm{~cm})$. There were also the following cases where the effects approached, but did not quite achieve, significance $(0.050 \leq p \leq 0.100)$ : (a) main effect of the recovery on the Pmean $\left(p=0.100, \eta^{2}=0.227\right.$, 383 versus $391 \mathrm{~W}$ in the $2 \mathrm{~min}$ and 5 min recovery, respectively); (b) main effect of the recovery on the $\mathrm{SJ}$ ( $\mathrm{p}=0.071$, $\eta^{2}=0.266,21.9$ versus $21.1 \mathrm{~cm}$ in the $2 \mathrm{~min}$ and $5 \mathrm{~min}$ recovery, respectively); and (c) main effect of the recovery on the EUR ( $p=0.082, \eta^{2}=0.249,1.044$ versus 1.089 in the $2 \mathrm{~min}$ and $5 \mathrm{~min}$ recovery, respectively). No differences were found in the lactate concentration, examined $5 \mathrm{~min}$ after the end of the protocol, between the two recovery conditions $\left(6.7 \pm 1.8\right.$ versus $6.0 \pm 1.6 \mathrm{mmol} \cdot \mathrm{L}^{-1}$ in the 2 and 5 min recovery, Cohen's $\mathrm{d}=0.4$ ).

\section{Discussion}

The main finding of the present study was that using a recovery of 2 or 5 min on a $5 \times 6 \mathrm{~s}$ RSE elicited similar physiological responses (for power output, changes in jumping performance and lactate concentration) in child soccer players. Another major finding was a main effect of the trial on the CMJ, which increased from the first trial to the third trial.

\section{Effect of the recovery on the power output}

The participants produced a similar power output, when either the peak or the mean values were considered, between the two recovery conditions. This finding was in agreement with a previous study using a $10 \times 10 \mathrm{~s}$ protocol with $30 \mathrm{~s}, 1 \mathrm{~min}$ and $5 \mathrm{~min}$ recoveries, which did not find any differences in the power output among the different recovery durations in 9.6 yrs boys. However, this was unlike post-pubescent and adult participants who needed the longest recovery duration in order to maintain their power output [27]. The similar power output between the 2 and 5 min recovery indicated a similar development of fatigue. The intramuscular accumulation of metabolic by-products, such as hydrogen ions, which accompany lactate production during RSE has been previously identified as a source of fatigue [14]. Since the participants showed similar levels of post-exercise lactate concentrations after both recovery conditions, it might be concluded that a similar fatigue mechanism was developed in both conditions.

\section{Effect of the trial on the jumping performance}

The height of the CMJ increased from the first trial to the third trial by $+1.5 \mathrm{~cm}(\sim 7 \%)$. Post-activation potentiation has been defined as an increase in the force production at sub-maximal levels of activation, due to the activation of fast-twitch skeletal muscle fibres [5] or the enhancement of the forces seen after the repetitive activation of 

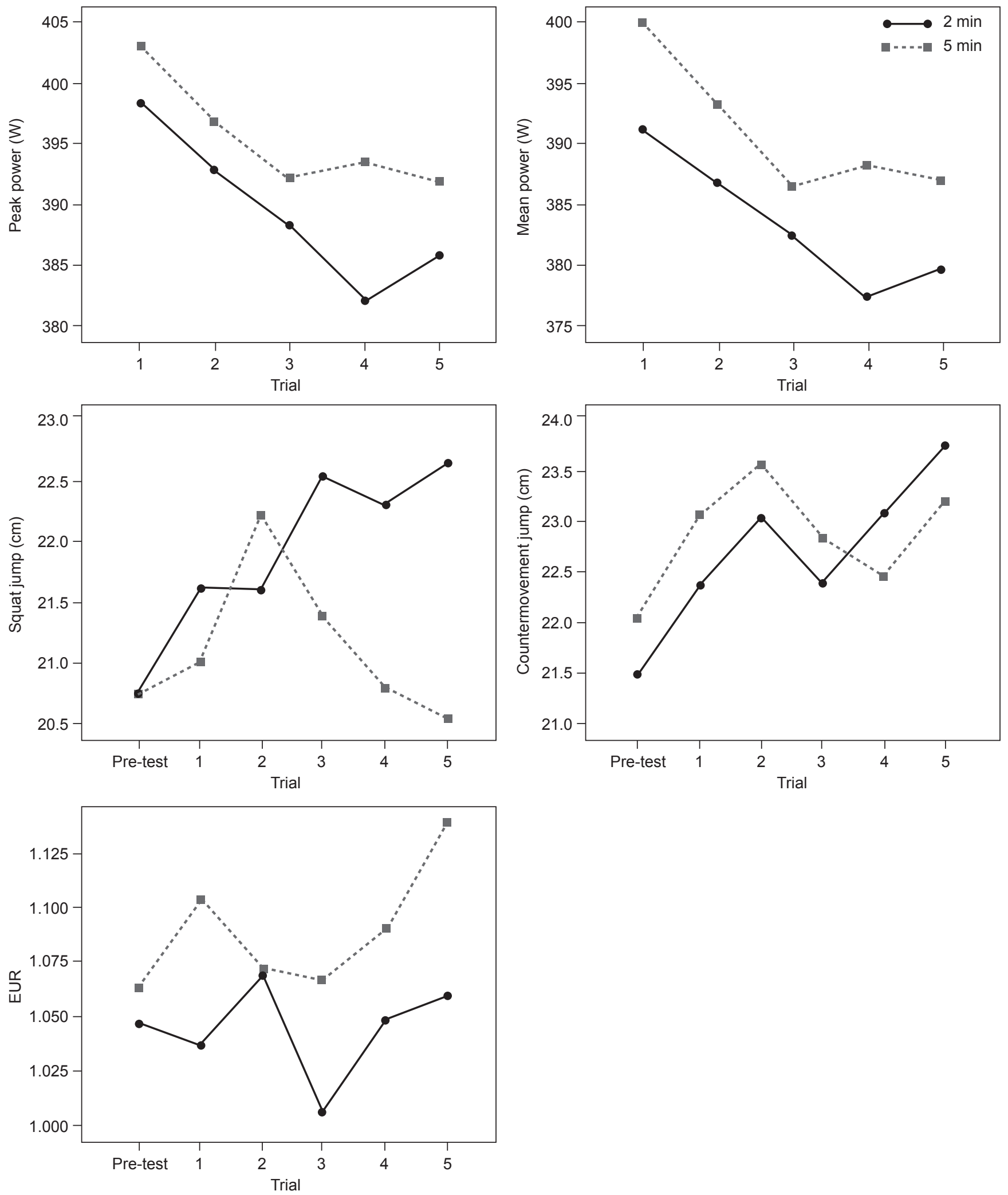

Fig. 1. Changes in peak power, mean power, height of squat jump and countermovement jump, and eccentric utilization ratio (EUR) 
the skeletal muscles [2]. On the other hand, fatigue is considered to reflect the inability of the skeletal muscles to generate an expected level of force, and the measured performance following muscle activity is the net balance between the fatigue and the post-activation potentiation [15]. Hence, there was a positive effect of the repeated sprints until the third trial.

\section{Effect of the trial on the power output}

Compared to the first trial, the Ppeak and Pmean decreased in the last trial by 2.9 and $3.1 \%$, respectively; however, these changes were not statistically significant. The changes of $\sim 3 \%$ were in agreement with existing studies. For instance, in a comparison of different recovery durations among $10 \times 5 \mathrm{~s}$ RSE, the peak power from the first trial to the last trial decreased by 8.5, 2.7 and 2.1\% with 25,50 and $100 \mathrm{~s}$ recoveries, respectively [24]. Also, a decrease of $13.2 \%$ from the first to the fifth trial was observed in a study of $5 \times 6 \mathrm{~s}$ RSE with a 24 s recovery [16]. Therefore, the use of a long recovery in the RSE with short sprints (e.g. 5 or $6 \mathrm{~s}$ ) resulted in a smaller decrease in the power output when compared to the shorter recovery.

\section{Limitations}

The present study was realised with $\sim 12$ yrs boys who were pre-pubescent according to their $\triangle \mathrm{APHV}(-2.3 \mathrm{yrs})$. A previous study compared the physiological responses to a $10 \times 10 \mathrm{~s}$ protocol with various recovery durations $(30 \mathrm{~s}$, $1 \mathrm{~min}$ and $5 \mathrm{~min}$ ) with participants of varying ages and found differences in the responses between $9.6 \mathrm{yrs}$ and 15.0 yrs boys [27]. Therefore, caution should be exercised when generalising the findings of the present study to other age groups. Another limitation of the findings might be the workload against which the participants pedalled, because it has been shown that the power output can vary according to the workload [4]. Also, the present study was a pilot one and the number of participants $(n=12)$, despite not differing from other studies comparing varying recovery durations, might be the reason that in three parameters (Pmean, SJ and EUR) the comparison findings approached, but did not quite achieve, significance. Thus, the use of a larger sample is recommended in future relevant research.

\section{Practical implications}

RSE consisting of a number of sprints, each lasting $\sim 6 \mathrm{~s}$, has been used routinely by sport scientists and practitioners. Since the exercise intensity - in addition to the workload, duration and number of repetitions - is also defined by the recovery, it would be of great practical importance for practitioners to be informed about the effect of recoveries of different durations. Since the two recovery times in the present study did not differ with regards to their physiological impact, the selection of the shorter recovery might contribute to a better management of the training session's duration. The exercise with a 2 min recovery lasted (4 recovery intervals among the trials $\times(5-2) \mathrm{min}) 12$ min less than the exercise protocol with the 5 min recovery, and this gain in time might be allocated to other purposes. As the additional time did not facilitate the recovery process, the selection of the shorter recovery might contribute to more time-savings and a more effective training session.

\section{Conclusions}

The duration of the recovery ( $2 \mathrm{~min}$ versus $5 \mathrm{~min}$ ) in the trials of repeated sprints did not induce important changes either to the parameters of the stretch-shortening cycle or to the power output of the participants. Based on these findings, it is recommended that fitness coaches use a 2 min recovery instead of a 5 min recovery when prepubescent athletes are performing sprints of a similar repetition rate and duration as in this study.

\section{References}

1. Argus C.K., Driller M.W., Ebert T.R., Martin D.T., Halson S.L. (2013) The effects of 4 different recovery strategies on repeat sprint-cycling performance. Int. J. Sports Physiol. Perform., 8(5): 542-548.

2. Binder-Macleod S.A., Dean J.C., Ding J. (2002) Electrical stimulation factors in potentiation of human quadriceps femoris. Muscle Nerve., 25(2): 271-279. DOI: 10.1002/mus. 10027.

3. Bogdanis G.C., Nevill M.E., Boobis L.H., Lakomy H.K.A., Nevill A.M. (1995) Recovery of power output and muscle metabolites following $30 \mathrm{~s}$ of maximal sprint cycling in man. J. Physiol., 482(2): 467-480.

4. Bogdanis G.C., Papaspyrou A., Theos A., Maridaki M. (2007) Influence of resistive load on power output and fatigue during intermittent sprint cycling exercise in children. Eur. J. Appl. Physiol., 101(3): 313-320. DOI: 10.1007/s00421-007-0507-7.

5. Brown I.E., Loeb G.E. (1999) Measured and modeled properties of mammalian skeletal muscle. I. The effects of post-activation potentiation on the time course and velocity dependencies of force production. J. Muscle Res. Cell Motil., 20(5-6): 443-456. DOI: 10.1023/ A:1005590901220.

6. Carlson J.S., Naughton G. (1994) Performance characteristics of children using various braking resistances on the wingate anaerobic test. J. Sports Med. Phys. Fitness, 34(4): 362-369. 
7. Cox G., Jenkins D.G. (1994) The physiological and ventilatory responses to repeated $60 \mathrm{~s}$ sprints following sodium citrate ingestion. J. Sports Sci., 12(5): 469-475. DOI: 10.1080/02640419408732197.

8. Dawson B., Fitzsimons M., Ward D. (1993) The relationship of repeated sprint ability to aerobic power and performance measures of anaerobic work capacity and power. Aust. J. Sci. Med. Sport, 25(4): 88-93.

9. Dawson B., Goodman C., Lawrence S., Preen D., Polglaze T., Fitzsimons M., Fournier P. (1997) Muscle phosphocreatine repletion following single and repeated short sprint efforts. Scand. J. Med. Sci. Sports, 7(4): 206-213.

10. Doré E., Bedu M., França N.M., Diallo O., Duché P., Van Praagh E. (2000) Testing peak cycling performance: Effects of braking force during growth. Med. Sci. Sports Exerc., 32(2): 493-498.

11. Driss T., Vandewalle H. (2013) The measurement of maximal (anaerobic) power output on a cycle ergometer: a critical review. Biomed Res. Int., 2013: 589361. DOI: 10.1155/2013/589361.

12. Engel F.A., Sperlich B., Stockinger C., Hartel S., Bos K., Holmberg H.C. (2015) The kinetics of blood lactate in boys during and following a single and repeated all-out sprints of cycling are different than in men. Appl. Physiol., Nutr. Metab., 40(6): 623-631. DOI: 10.1139/apnm2014-0370.

13. Fernandez-Santos J.R., Ruiz J.R., Cohen D.D., GonzalezMontesinos J.L., Castro-Piñero J. (2015) Reliability and Validity of Tests to Assess Lower-Body Muscular Power in Children. J. Strength Cond. Res., 29(8): 2277-2285. DOI:10.1519/JSC.0000000000000864.

14. Girard O., Mendez-Villanueva A., Bishop D. (2011)Repeated-sprint ability part I: Factors contributing to fatigue. Sports Med., 41(8): 673-694. DOI: 10.2165/11590550000000000-00000.

15. Hodgson M., Docherty D., Robbins D. (2005) Postactivation potentiation: Underlying physiology and implications for motor performance. Sports Med., 35(7): 585-595. DOI:10.2165/00007256-200535070-00004.

16. Jaafar H., Rouis M., Coudrat L., Gélat T., Noakes T.D., Driss T., Eynon N. (2015) Influence of affective stimuli on leg power output and associated neuromuscular parameters during repeated high intensity cycling exercises. PLoS ONE 10(8). DOI:10.1371/journal.pone.0136330.

17. Jones B., Cooper C.E. (2014) Use of NIRS to assess effect of training on peripheral muscle oxygenation changes in elite rugby players performing repeated supramaximal cycling tests. In: Advances in Experimental Medicine and Biology, pp. 333-339.

18. Lee C.L., Cheng C.F., Lin J.C., Huang H.W. (2012) Caffeine's effect on intermittent sprint cycling performance with different rest intervals. Eur. J. Appl. Physiol., 112(6): 2107-2116. DOI: 10.1007/s00421-011-2181-z.
19. Linthorne N.P. (2001) Analysis of standing vertical jumps using a force platform. American Journal of Physics 69(11): 1198-1204. DOI: 10.1119/1.1397460.

20. Lopez E.I.D., Smoliga J.M., Zavorsky G.S. (2014) The effect of passive versus active recovery on power output over six repeated Wingate sprints. Res. Q. Exerc. Sport, 85(4): 519-526. DOI: 10.1080/02701367.2014.961055.

21. Matsuura R., Arimitsu T., Yunoki T., Kimura T., Yamanaka R., Yano T. (2015) Effects of heat exposure in the absence of hyperthermia on power output during repeated cycling sprints. Biol. Sport, 32(1): 15-20. DOI: $10.5604 / 20831862.1125286$.

22. Mirwald R.L., Baxter-Jones A.D., Bailey D.A., Beunen G.P. (2002) An assessment of maturity from anthropometric measurements. Med. Sci. Sports Exerc., 34(4): 689-694.

23. Nikolaidis P.T., Dellal A., Torres-Luque G., Ingebrigtsen J. (2015) Determinants of acceleration and maximum speed phase of repeated sprint ability in soccer players: A cross-sectional study. Sci. Sports, 30(1): e7-e16. DOI: 10.1016/j.scispo.2014.05.003.

24. Ohya T., Aramaki Y., Kitagawa K. (2013) Effect of duration of active or passive recovery on performance and muscle oxygenation during intermittent sprint cycling exercise. Int. J. Sports Med., 34(7): 616-622. DOI: 10.1055/ s-0032-1331717.

25. Pearcey G.E.P., Murphy J.R., Behm D.G., Hay D.C., Power K.E., Button D.C. (2015) Neuromuscular fatigue of the knee extensors during repeated maximal intensity intermittent-sprints on a cycle ergometer. Muscle Nerve, 51(4): 569-579. DOI:10.1002/mus.24342.

26. Rakobowchuk M., Tanguay S., Burgomaster K.A., Howarth K.R., Gibala M.J., MacDonald M.J. (2008) Sprint interval and traditional endurance training induce similar improvements in peripheral arterial stiffness and flow-mediated dilation in healthy humans. Am. J. Physiol. Regul, Integr, Comp, Physiol., 295(1): R236-R242. DOI: 10.1152/ajpregu.00069.2008.

27. Ratel S., Bedu M., Hennegrave A., Doré E., Duché P. (2002) Effects of age and recovery duration on peak power output during repeated cycling sprints. Int. J. Sports Med., 23(6): 397-402. DOI: 10.1055/s-2002-33737.

28. Ratel S, Duche P, Hennegrave A, Van Praagh E, Bedu M. (2003) Acid-base balance during repeated cycling sprints in boys and men. J. Appl. Physiol., 92(2): 479-485.

29. Ross W.D., Marfell-Jones M.J. (1991) Kinanthropometry. In: J.D. MacDougall, H.A. Wenger and H.J. Green (eds.) Physiological testing of the high-performance athlete. Champaign, IL: Human Kinetics.

30. Shepherd S.O., Wilson O.J., Taylor A.S., ThøgersenNtoumani C., Adlan A.M., Wagenmakers A.J.M., Shaw C.S. (2015) Low-volume high-intensity interval training in a gym setting improves cardio- 
metabolic and psychological health. PLoS ONE 10(9). DOI: 10.1186/2008-2231-22-43.

31. Spencer M., Bishop D., Dawson B., Goodman C. (2005) Physiological and metabolic responses of repeated-sprint activities: Specific to field-based team sports. Sports Med., 35(12): 1025-1044. DOI: 10.2165/00007256200535120-00003.

32. Townsend J.R., Stout J.R., Morton A.B., Jajtner A.R., Gonzalez A.M., Wells A.J., Mangine G.T., McCormack W.P., Emerson N.S., Robinson IV E.H., Hoffman J.R., Fragala M.S., Cosio-Lima L. (2013) Excess post-exercise oxygen consumption (EPOC) following multiple effort sprint and moderate aerobic exercise. Kinesiol., 45(1): 16-21.

33. Welsh A.H., Knight E.J. (2014) "Magnitude-based Inference": A statistical review. Med. Sci. Sports Exerc., 47(4): 874-884. DOI: 10.1249/MSS.0000000000000451.
34. Whyte L.J., Gill J.M.R., Cathcart A.J. (2010) Effect of 2 weeks of sprint interval training on health-related outcomes in sedentary overweight/obese men. Metab. Clin. Exp., 59(10): 1421-1428. DOI: 10.1016/j. metabol.2010.01.002.

\section{Received 05.02.2016 \\ Accepted 02.05.2016}

(C) University of Physical Education, Warsaw, Poland

\section{Acknowledgments}

The voluntarily participation of the children soccer players and the cooperation with their coach and parents is gratefully acknowledged. 